\section{GP281 TRENDS IN ANTIBIOTIC TREATMENT CHANGES OF HOSPITALIZED COMMUNITY-ACQUIRED PNEUMONIA IN CHILDREN}

${ }^{1}$ Emilija Tamelyte, ${ }^{1}$ Ruta Ceidaite, 1,2Laimute Vaideliene, 1,2 Lina Jankauskaite*. 'Lithuanian University of Health Sciences, Kaunas, Lithuania; ${ }^{2}$ Lithuanian University of Health Sciences Hospital Kauno Klinikos, Department of Pediatrics, Kaunas, Lithuania

\subsection{6/archdischild-2019-epa.340}

Community-acquired pneumonia (CAP) is a leading cause of death in children worldwide. Failure to choose correct antibiotic, dose or treatment duration worsens CAP outcomes and contributes to global antibiotic $(\mathrm{AB})$ resistance.

Aim To analyze and compare antimicrobial treatment among children with hospitalized CAP in year 2012 and 2017.

Methods Retrospective analysis of previously healthy children with hospitalized CAP in 2012( $(n=85)$ and 2017( $n=96)$. All cases were divided into CAP during flu and non-flu seasons and subdivided according to age ( $<2 \mathrm{mo}, 2-12 \mathrm{mo}, 1-5 \mathrm{y},>5 \mathrm{y})$. Statistical analysis was performed with Microsoft Excel and IBM SPSS Statistics software. P value $<0.05$ was considered significant.

Results There was no difference in children age, gender and duration of hospitalization between all groups. Most of the cases were hospitalized during the flu season $(68 \%$ vs $77 \%$ in 2012 and 2017 respectively). Significant change in first-line antibiotic therapy was found. The most frequent antibiotics to treat hospitalized CAP in children in 2012 were Penicillin (PEN) (59\%), Cefuroxime (CXM) (28\%) and Clarithromycin (CLR) (12\%). Ampicillin-sulbactam (SAM) was started in 35\% of children in 2017 and younger children were more likely to receive it $(p=0.047)$. Decrease in first-line treatment with CXM and PEN was observed in $2017(8 \%$ and $38 \%$ respectively). Only $12 \%$ of children received CLR in 2017. Initial dose of PEN was $0.117 \pm 0.026 \mathrm{mioU} / \mathrm{kg}$ in 2012 with slight increase in dosage of $0.125 \pm 0.022 \mathrm{mioU} / \mathrm{kg}$ in $2017(\mathrm{p}=0.07)$ with tendency of higher doses in flu season $(p=0.0564)$. Significant elevation was observed in dosing CLR (11.49 \pm 3.62 $\mathrm{mg} / \mathrm{kg}$ (2012), $17.47 \pm 5.24 \mathrm{mg} / \mathrm{kg}$ (2017), $\mathrm{p}=0.0344)$. It was significantly higher comparing between flu seasons $(\mathrm{p}=0.0128)$. $\mathrm{AB}$ were changed, dosage increased, or another antibiotic added in 27 cases in 2012, 85\% of which were during flu season. CXM was the most frequently used as secondline antibiotic in 2012. Its dose was adjusted, or it was changed in 15 cases and $87 \%$ of changes were made during flu season. In 2017, 27 children received $A B$ adjustment with $63 \%$ of changes during flu season. Primary antibiotic was shifted to SAM in $65 \%(n=17)$ of CAP in 2017 and $71 \%$ of the changes during flu season.

Conclusion There was significant change in first-line antibiotic between 2012 and 2017. CAP treatment in 2017 was started with SAM in $35 \%$ of the cases and it was most frequently chosen as second-line antibiotic. Higher dosage of antibiotics and antibiotical adjustment in 2012 and 2017 was associated with flu season.

\section{GP282 AN EVALUATION OF THE NORMAL RANGES OF EXERCISE TOLERANCE IN HEALTHY CHILDREN AND ADOLESCENTS AS MEASURED BY THE MODIFIED SHUTTLE TEST (MST)}

'LM Corley*, ${ }^{2} \mathrm{~S}$ Canny, ${ }^{2} \mathrm{~L}$ O'Connor, ${ }^{2} \mathrm{M}$ Lynn, ${ }^{2} \mathrm{MB}$ O'Neill. 'University College Dublin, Dublin, Ireland; ${ }^{2}$ Mayo University Hospital, Castlebar, Ireland

\subsection{6/archdischild-2019-epa.341}

Aim The aim of this study was to utilise the Modified Shuttle Test (MST) to determine the exercise capacity range of Irish children and adolescents 5 to 15 years of age. The MST is a well defined test, administered by a trained physiotherapist, following the protocol described by LaLeger.

Methods Children and Adolescents 5-15 years of age with negative bronchial reactivity testing and an absence of underlining chronic respiratory or cardiac disease were eligible for inclusion. The MST has 15 levels, each lasting 1 minute, with incremental increases in speed of $0.61 \mathrm{~km} / \mathrm{h}$ each minute. The test requires participants to move between two markers, which are 10 metres apart, in time with the beeps from a prerecorded digital audio track. The test is halted if the participant is unable to continue or fails to reach the 10 meter

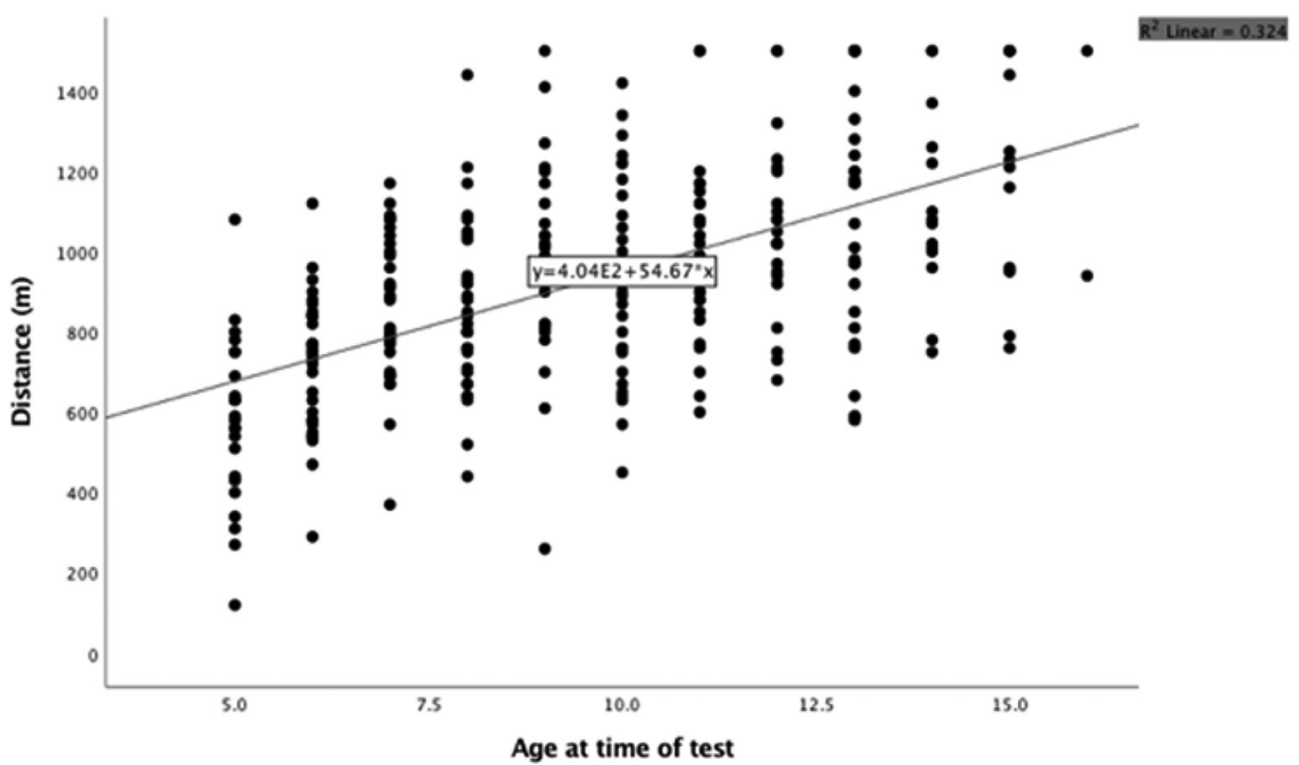

Abstract GP282 Figure 1 
mark on two consecutive beeps. The test takes on average 25 minutes to complete.

Results Data was available on 277 children with negative bronchial reactivity. The $\mathrm{M} ; \mathrm{F}$ was $1.27: 1$. The mean age was 10 years old (range 5-15). The number of patients per age in years ranged from 14 to 33 (mean 25). The line of best fit indicates that the mean exercise distance reached in meters was given by the formula $\mathrm{Y}=(54.7 \times$ Age $)+404$. The study noted that the main reasons for test cessation related to fatigue and shortness of breath.

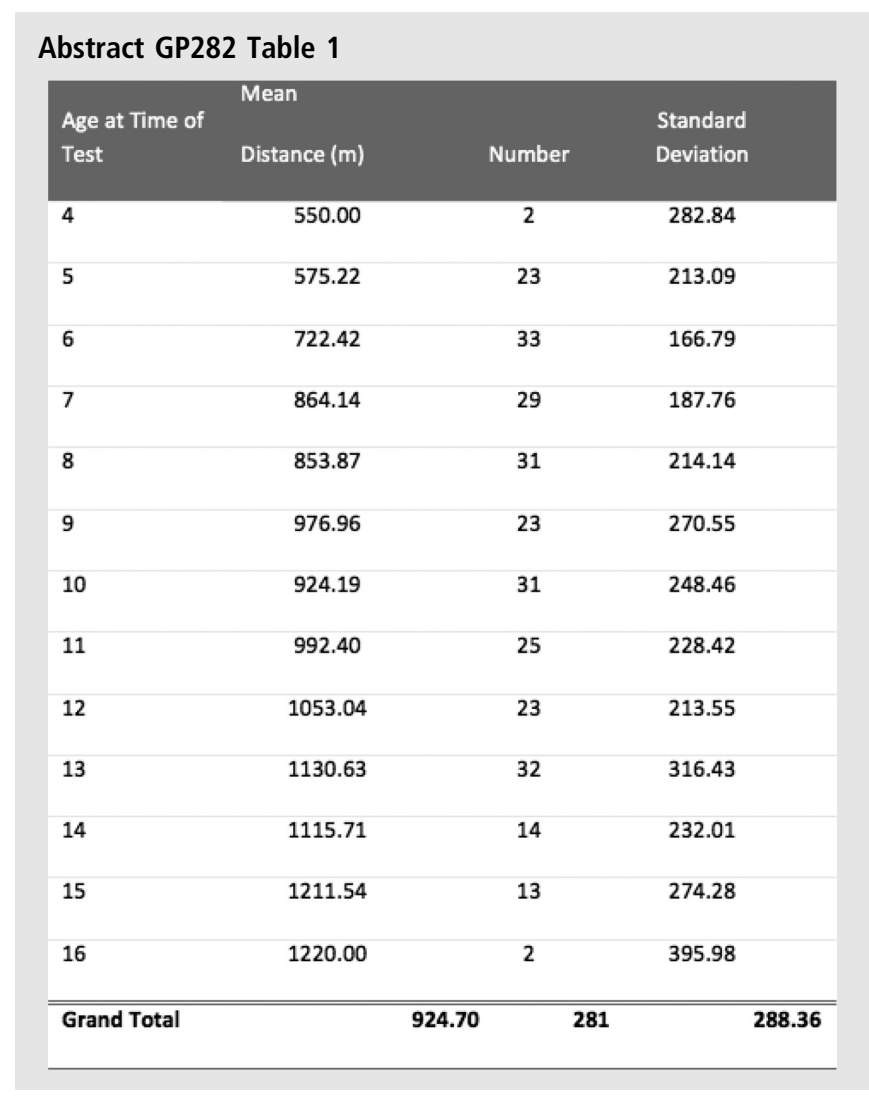

Conclusions This study defines normative data for children's exercise capacity as measured by the MST. It provides a frame of reference for parents in discussing their child's exercise tolerance and the modified formula is easy to use (distance travelled $=55 \mathrm{x}$ Age $+400 \mathrm{~m})$.

\section{GP283 CLINICAL AND RADIOLOGICAL EVALUATION OF PATIENTS WITH POSTINFECTIOUS BRONCHIOLITIS OBLITERANS}

${ }^{1}$ Erkan Cakir, ${ }^{1}$ Fatouma Khalif, ${ }^{2}$ Mehmet Bilgin, 'Lina Al Shadfan, ${ }^{1}$ Hakan Yazan, ${ }^{3}$ Irem Yilmaz*, ${ }^{4}$ Mustafa Atilla Nursoy. 'Bezmialem Vakif University, Faculty of Medicine, Department of Pediatric Pulmonology, Istanbul, Turkey; ${ }^{2}$ Bezmialem Vakif University, Faculty of Medicine, Department of Radiology, Istanbul, Turkey; ${ }^{3}$ Bezmialem Vakif University, Faculty of Medicine, Department of Pediatrics, Istanbul, Turkey; ${ }^{4}$ Bezmialem Vakif University, Faculty of Medicine, Department of Pediatric Allergy and Immunology, Istanbul, Turkey

\subsection{6/archdischild-2019-epa.342}

Introduction Postinfectious bronchiolitis obliterans is characterized by persistent symptoms and signs of obstructive pulmonary disease following acute bronchial injury. It usually occurs with persistent wheezing and cough as a result of severe viral infections. The aim of this study was to investigate the socio- demographic characteristics, treatment status, and clinical response of our cases of postinfectious bronchiolitis obliterans. Methods The records of 102 patients with postinfectious bronchiolitis obliterans who were followed-up in our clinic were retrospectively reviewed. Sociodemographic characteristics, treatments, pre-treatment and post-treatment complaints, pulmonary function tests, clinical status, radiological changes were evaluated.

Results 68.6\% ( $\mathrm{n}=70)$ of our patients were male. The mean age of the patients at the time of the study was $88.8 \pm 53.5$ months, and the age of diagnosis was $34.3 \pm 36.4$ months. The mean period from the first complaints to the date of diagnosis was $22.8 \pm 33.8$ months. 59\% (57) of the patients had a history of smoking in one of their parents. In $62.7 \%$ of the cases, the first lower respiratory tract infection was bronchopneumonia and $36.3 \%$ was in the acute bronchiolitis. Before the diagnosis, 35 patients had intensive care hospitalization (34.3\%). Persistent cough (90.2\%), wheezing (96.1\%), shortness of breath (91.2\%) and purulant cough (75\%) were the most frequent findings. When treatments of the patients were evaluated; inhaled corticosteroids were 92.2\%, 6 months azithromycin $69.6 \%, 6$ months oral steroid $61.8 \% .11$ patients $(10.8 \%)$ needed oxygen and 10 patients $(9.8 \%)$ required Bilevel Positive Airway Pressure (BPAP) support. Before and after treatment of 49 patients, lung CTs were evaluated by radiologist and modified Bhalla scores were calculated. Pre-treatment mosaism and peribronchial thickening were detected in all patients. There were bronchiectasis $67.3 \%$, atelectasis $91.8 \%$, hyperinflation $81.6 \%$, air trapping $98 \%$ of patients. Post-treatment radiological evaluation revealed mosaism 93.9\%, peribronchial thickening $100 \%$, bronchiectasis 51\%, atelectasis $65.3 \%$, hyperinflation $69.4 \%$, air trapping $87.8 \%$. The mean Bhalla score was $9.1 \pm 4.1$ before the treatment and it was found to be $6.8 \pm 4.6$ after treatment.

Discussion and Conclusion Postinfectious bronchiolitis obliterans is a rare condition with obstruction findings. It occurs as a result of immune mechanisms after severe lung infection, especially at a young age. In this study, we aimed to present sociodemographic characteristics, clinical features and especially response to long-term sterolid and azithromycin treatment in patients with bronchiolitis obliterans.

\section{GP284 THE EFFECTS OF IVACAFTOR ON PANCREATIC FUNCTION IN PAEDIATRIC PATIENTS WITH CYSTIC FIBROSIS GATING MUTATIONS}

Joshua Emery*, David Mullane, Muireann Ni Chroinin. University College Cork, Cork, Ireland

\subsection{6/archdischild-2019-epa.343}

Objectives To examine the effects of Ivacaftor on growth and pancreatic function in patients attending the Paediatric Cystic fibrosis (CF) Centre at Cork University Hospital.

Methods A retrospective, convenience sampled cohort analysis was conducted in patients aged 2 to 17 with genetically confirmed CF ( $\mathrm{N}=28,15$ male, 13 female). Subjects who received Ivacaftor over a 1 year period had an oral dose of either 75 or $150 \mathrm{mg}$ twice daily depending on weight. Patients were excluded if concurrently taking Lumacaftor. The primary end points were estimated mean change from baseline through one year in pancrealipase (Creon) consumption per day and body mass index (BMI). Secondary end points included the changes in mean percent of predicted forced 